\title{
6. Politische Partizipation: Unterschriftenaktionen, Demonstrationen, Bürgerinitiativen und politische Ämter
}

\author{
Julia Simonson \& Claudia Vogel
}

\section{Kernaussagen}

Bürgerinnen und Bürger nehmen in vielfältiger Weise am politischen Leben teil. Über den gesamten bisherigen Lebenslauf betrachtet sind verbreitete Formen der politischen Partizipation: die Teilnahme an Unterschriftensammlungen (43,4 Prozent), an Demonstrationen (32,2 Prozent) und an Bürgerinitiativen (22,5 Prozent) sowie die Übernahme von politischen Ämtern oder anderweitiger politischer Verantwortung (8,7 Prozent).

Frauen beteiligen sich bei allen politischen Partizipationsformen zu geringeren Anteilen als Männer. Dieser Geschlechterunterschied ist bei der Teilnahme an Demonstrationen und bei der Übernahme politischer Ämter besonders ausgeprägt.

Die Altersgruppe der 50- bis 64-Jährigen hat die höchsten Beteiligungsquoten an Unterschriftensammlungen, Demonstrationen und Bürgerinitiativen. Zudem haben 11,7 Prozent dieser Altersgruppe und 12,8 Prozent der Personen im Alter von 65 und mehr Jahren schon einmal ein politisches Amt ausgeübt oder anderweitig politische Verantwortung übernommen. Die entsprechenden Anteile bei den jüngeren Altersgruppen sind deutlich geringer.

Alle Partizipationsformen sind bei Personen mit hoher Schulbildung weiter verbreitet als bei Personen mit mittlerer oder niedriger Schulbildung. Während beispielsweise mehr als die Hälfte der Personen mit hoher Bildung schon einmal an einer Bürgerinitiative beteiligt war, hat sich von den Personen mit niedriger Bildung nur etwa jede beziehungsweise jeder Vierte schon einmal daran beteiligt.

Betrachtet man die vier Partizipationsformen gemeinsam, zeigt sich, dass sich insgesamt 58,2 Prozent aller in Deutschland lebenden Personen im Alter ab 14 Jahren auf mindestens eine Art und Weise beteiligt haben. Etwa ein Drittel aller Personen hat sich sogar schon auf verschiedene Arten und Weisen politisch eingebracht, mehr als jede beziehungsweise jeder Achte hat zudem drei und mehr Partizipationsformen genannt. Ein Viertel hat sich lediglich in einer Form beteiligt.

\subsection{Einleitung}

Politische Partizipation ist ein Grundbestandteil der Demokratie, für die zumindest ein minimales Niveau der Partizipation der Bürgerinnen und Bürger existenziell ist. „Wer Demokratie sagt, meint Partizipation“ (van Deth 2009: 6). In demokratischen Systemen haben die Bürgerinnen und Bürger das Recht, aktiv am politischen Leben teilzunehmen (Gabriel 2014). Politische Partizipation bezeichnet dabei alle freiwilligen Aktivitäten, mit denen Bürgerinnen und Bürger

(C) Der/die Autor(en) 2017

J. Simonson et al. (Hrsg.), Freiwilliges Engagement in

Deutschland, Empirische Studien zum bürgerschaftlichen

Engagement, DOI 10.1007/978-3-658-12644-5_7 
außerhalb ihres Berufes versuchen, Einfluss auf politische Entscheidungen zu nehmen oder an diesen mitzuwirken (Gabriel 2014: 28). Aus demokratietheoretischer Sicht liegt die Hauptmotivation für politische Partizipation also im Gestaltungswillen (Roth 2011). Die Einflussnahme der Bürgerinnen und Bürger kann dabei sehr unterschiedlich ausgestaltet sein, es kann sich um punktuelle Einzelaktivitäten oder um dauerhafte politische Partizipation handeln. Neben institutionellen Formen der Beteiligung, wie der Teilnahme an Wahlen oder der Mitarbeit in Parteien können Bürgerinnen und Bürger auch nicht-institutionalisierte Formen der Beteiligung wie Unterschriftensammlungen, Demonstrationen und Bürgerinitiativen nutzen (Weßels 2013). In der Regel berufen sich auch Personen, die nicht-institutionalisierte Partizipationsformen ausüben, auf verfassungsmäßig garantierte politische Mitwirkungsrechte wie die Versammlungs- und Vereinigungsfreiheit (Roth 2011).

Nach Kersting lassen sich vier Arten der politischen Beteiligung unterscheiden: Wahlen als Form der repräsentativen Demokratie, Volksentscheide und Bürgerinitiativen als Form der direkten Demokratie, Demonstrationen und Unterschriftenaktionen als demonstrative Demokratie sowie moderne Beiräte als deliberative, also beratende, Demokratie. Letzteres bedeutet, dass Bürgerinnen und Bürger an politischen Planungs- und Entscheidungsprozessen beteiligt werden, indem sie zum Zwecke der öffentlichen Beratung und Konsensfindung konsultiert werden und so an Entscheidungen mitwirken (Kersting 2013). Durch die frühzeitige Beteiligung der Bürgerinnen und Bürger soll sich die Akzeptanz der Entscheidungen zu bestimmten öffentlichen Vorhaben erhöhen. Neue technische Möglichkeiten durch die Nutzung des Internets machen nicht nur die Durchführung einer Online-Petition, sondern auch die direkte Bürgerbeteiligung deutlich einfacher, wie zahlreiche Internetplattformen veranschaulichen. Solche Bürgerbeteiligungsplattformen lassen sich in fast allen Kommunen Deutschlands ebenso finden wie zahlreiche Beispiele für aktuelle Be- teiligungsprozesse: Stellvertretend seien hier die Bürgerbeteiligung zum Innenstadtverkehrskonzept Potsdam ${ }^{1}$, zum Energiekonzept der Landeshauptstadt Stuttgart ${ }^{2}$ und zum Spielplatz Riemer Feld in München ${ }^{3}$ genannt. Die Ausweitung der Instrumente im Bereich der deliberativen Demokratie kann zum Anstieg des freiwilligen Engagements in Deutschland beitragen (siehe Kapitel 3).

In diesem Kapitel wird der Frage nachgegangen, wer sich in Deutschland in unterschiedlichen Partizipationsformen politisch beteiligt. Konkret betrachten wir vier verschiedene Formen der politischen Partizipation: Die Teilnahme an Unterschriftensammlungen oder Online-Petitionen betrachten wir ebenso wie die Beteiligung an Demonstrationen, die beide der demonstrativen Demokratie sowie den nicht-institutionalisierten Formen zuzurechnen sind. Zudem betrachten wir die Bürgerinitiativen als Form der direkten Demokratie, wobei Bürgerinitiativen wiederum vielfältig ausgestaltet sein können, und es sich bei dem Begriff sowohl um eine Selbst- als auch um eine Fremdbezeichnung handeln kann (Wagner 2013). Im Falle, dass Bürgerinitiativen auch in die politischen Planungsprozesse einbezogen werden, können sie teils auch als Form der deliberativen Demokratie betrachtet werden, und sie zählen ebenfalls zu den nicht-institutionalisierten Formen. Diese Formen zeichnen sich mehrheitlich durch einen vergleichsweise eher geringen Verpflichtungsgrad aus, vielleicht mit Ausnahme der in diesen Feldern freiwillig engagierten Organisatoren und Initiativgeber. Anschließend betrachten wir die Übernahme eines politischen Amtes oder einer sonstigen politischen Verpflichtung als institutionalisierte Form der Partizipation, die zumindest im Fall des Wahlamtes der direkten Demokratie zuzuordnen ist. Nicht-institutionalisierte Formen der

1 http://buergerbeteiligung.potsdam.de/content/innenstadtverkehrskonzept

2 www.stuttgart-meine-stadt.de/content/bbv/details/44/

3 http://www.muenchen.de/rathaus/Stadtverwaltung/ baureferat/buergerschaftliches-engagement/beteiligung-spielplatz.html 
Partizipation wie Bürgerinitiativen galten lange Zeit als ,unkonventionelle 'Möglichkeiten sich einzubringen, seit den 1970er Jahren haben sie sich im Zuge der ,partizipatorischen Revolution jedoch weitgehend etabliert (Weßels 2013).

Politische Themen, welche die Menschen bewegen, werden heute meist durch verschiedene Partizipationsmöglichkeiten begleitet. Am Beispiel der geplanten Bebauung des Tempelhofer Feldes in Berlin lässt sich dies verdeutlichen: Es wurden Unterschriften gesammelt und ein Volksentscheid erzwungen, es gab mehrere Demonstrationen und Aktionen auf dem Tempelhofer Feld, um auf die Positionen der verschiedenen Beteiligten aufmerksam zu machen, es wurden Bürgerinitiativen wie der Verein ,Demokratische Initiative 100\% Tempelhofer Feld ${ }^{4}$ gegründet. Politische Amtsträger sowohl in der Bezirksverwaltung als auch in der Senatsverwaltung, aber auch in den Vereinen und Aktionsbündnissen sind bis heute in die weitere Planung für das Tempelhofer Feld involviert. Zudem werden die Bürgerinnen und Bürger über die Öffentlichkeitsbeteiligung in die weitere Planung eingebunden. ${ }^{5}$

Manche der in diesem Kapitel untersuchten politischen Aktivitäten fallen unter die Definition des freiwilligen Engagements, etwa wenn es sich um die unentgeltliche Übernahme eines politischen Amtes handelt (siehe Kapitel 1). Andere lassen sich dem weiter gefassten Konzept des zivilgesellschaftlichen Handelns zuordnen, welches sich durch ein Mitmachen außerhalb von Beruf und Familie auszeichnet. Eine Meinungsäußerung oder eine Interessenvertretung wie das Unterschreiben einer Petition oder das Demonstrieren sind ebenso als zivilgesellschaftliches Handeln $\mathrm{zu}$ verstehen wie etwa die informelle Unterstützung von Nachbarinnen und Nachbarn (siehe Kapitel 9). Die manchmal etwas unscharfe Trennlinie zwischen freiwilligem Engagement und anderen Formen zivilgesellschaftlichen

4 www.thf100.de

5 www.stadtentwicklung.berlin.de/umwelt/stadtgruen/ tempelhofer_feld
Handelns kann am Beispiel der Bürgerinitiative aufgezeigt werden. Eine Initiative braucht Personen, die diese organisieren und das Vorhaben voranbringen, aber auch Unterstützerinnen und Unterstützer, die beispielsweise an von der Initiative angemeldeten Demonstrationen oder Versammlungen teilnehmen, aber selbst keine freiwilligen Aufgaben wie das Organisieren von Veranstaltungen übernehmen und daher nicht freiwillig engagiert sind. Das bedeutet, alle Personen, die sich im Bereich der politischen Partizipation freiwillig engagieren, sind auch zivilgesellschaftlich aktiv, aber nicht alle zivilgesellschaftlich aktiven Personen sind freiwillig engagiert.

An welche individuellen Voraussetzungen ist die politische Partizipation geknüpft? Notwendig ist das Interesse für politische Belange sowie die Überzeugung, selbst etwas politisch verändern zu können. Personen müssen außerdem einen Gestaltungs- beziehungsweise Mitbestimmungswillen haben (Bödeker 2012a). Darüber hinaus setzt politische Partizipation voraus, auf mehr oder weniger umfangreiche individuelle Ressourcen zurückgreifen zu können. Personen müssen beispielsweise wissen, wo und wie sie ihre Interessen einbringen können, sie müssen die notwendigen sozialen Kontakte haben oder diese knüpfen können, und es müssen genügend zeitliche und teilweise auch finanzielle Ressourcen vorhanden sein. An die politische Partizipation sind also ganz ähnliche Voraussetzungen geknüpft wie an andere Formen der gesellschaftlichen Teilhabe. Auch die politische Partizipation variiert stark mit Einkommen und Bildung, wobei Personen mit höherem Einkommen und höherer Bildung im Durchschnitt auch höhere Partizipationsraten zeigen (Bödeker 2012b).

Neben solchen Bildungsunterschieden werden in der Literatur auch Geschlechterunterschiede diskutiert, wobei Frauen etwas geringere Partizipationsraten aufweisen als Männer (Westle 2001). Die Gründe, die hierfür angeführt werden können, sind vielfältig: So verfügen Frauen durchschnittlich seltener über partizipationsrelevante Voraussetzungen wie beispielsweise 
entsprechende, durch die Erwerbstätigkeit vermittelte, soziale Kontakte als Männer. Darüber hinaus kann die stärkere zeitliche Beanspruchung von Frauen im Sinne einer Doppelbelastung durch Familie und Beruf eine Rolle spielen. Zusätzlich können insbesondere bei älteren Geburtsjahrgängen Unterschiede in der Sozialisation bedeutsam sein, die Frauen traditionell die Rolle der helfenden Person und Männern die Rolle der politisch aktiven und mächtigen Person zudachte (Cornelissen 1993). Schließlich ist es möglich, dass insbesondere bei institutionalisierten Formen der politischen Beteiligung Frauen nach wie vor stärkere Vorbehalte entgegengebracht werden als Männern, sodass es für Frauen schwieriger ist, mit gleicher Selbstverständlichkeit in der Politik zu agieren wie Männer (Westle 2001: 164).

Darüber hinaus wurde davon ausgegangen, dass Jüngere stärker als Ältere zu nicht-institutionalisierten, alternativen Partizipations- und Protestformen neigen. Neuere Untersuchungen zeigen jedoch, dass die Beteiligung Jüngerer an Unterschriftensammlungen, Demonstrationen und Bürgerinitiativen nicht höher ist als im Bevölkerungsdurchschnitt - diese Formen der Partizipation gehören mittlerweile zu den etablierten politischen Beteiligungsformen aller Bürgerinnen und Bürger (Weßels 2013: 365).

Im Folgenden soll beantwortet werden, $\mathrm{zu}$ welchen Anteilen welche Bevölkerungsgruppen in unterschiedlichen Formen partizipieren. Im Einzelnen wird diesen Fragen nachgegangen:

\subsection{Datengrundlage}

Die in diesem Kapitel berichteten Befunde basieren auf gewichteten Analysen. Für die Gewichtung wurden in allen Erhebungsjahren dieselben Gewichtungsmerkmale berücksichtigt. Dabei handelt es sich um die Designgewichtung, die sich auf die Auswahlwahrscheinlichkeit einer Person bezieht (Haushaltsgröße, Zahl der Festnetznummern und, nur für das Jahr 2014,

auch die Zahl der Mobilfunknummern) und Gewichtungsmerkmale, die Abweichungen der Stichprobenverteilung von der Grundgesamtheit in bestimmten Dimensionen ausgleichen (Bundesland, Gemeindegrößenklassen, Geschlecht, Altersgruppen). Von einer Hinzunahme weiterer Gewichtungsmerkmale wie etwa des Bildungs-

1. Wie hoch ist der Anteil der Menschen, die sich schon einmal an einer Unterschriftensammlung beziehungsweise einer Online-Petition beteiligt haben? Wie unterscheiden sich Frauen und Männer, Jüngere und Ältere sowie unterschiedliche Bildungsgruppen hinsichtlich der Beteiligung?

2. Wie hoch ist der Anteil derer, die schon einmal an einer Demonstration teilgenommen haben? Welche Unterschiede in der Beteiligung zeigen sich nach Geschlecht, Alters- und Bildungsgruppen?

3. Wie hoch ist der Anteil der Personen, die schon einmal bei einer Bürgerinitiative mitgemacht haben? Wie unterscheidet sich die Teilnahme an einer Bürgerinitiative nach Geschlecht, nach Altersgruppen sowie nach Bildung?

4. Wie hoch ist der Anteil derer, die schon einmal ein politisches Amt oder anderweitig politische Verantwortung übernommen haben? Welche Unterschiede zeigen sich nach Geschlecht, Alters- und Bildungsgruppen?

5. Wie hoch ist der Anteil derer, die sich lediglich in einer oder aber in mehreren Formen politisch beteiligt haben?

\footnotetext{
standes haben wir abgesehen, um die Vergleich-
} 
barkeit mit den bisher veröffentlichten Berichten zu erhalten.

Im Deutschen Freiwilligensurvey 2014 wurden vier Formen der politischen Partizipation mit unterschiedlichem Verpflichtungsgrad erhoben:

Es gibt verschiedene Möglichkeiten, politisch aktiv $z u$ sein. Welche der folgenden Dinge haben Sie bereits getan? Haben Sie schon einmal ein politisches Amt oder anderweitig politische Verantwortung übernommen? Haben Sie schon einmal bei Unterschriftensammlungen oder Online-Petitionen für politische Ziele unterschrieben? Haben Sie schon einmal an einer Demonstration teilgenommen? Haben Sie schon einmal bei einer Bürgerinitiative mitgemacht?

Die Antwortmöglichkeiten lauteten jeweils ,ja und ,nein', Mehrfachnennungen waren möglich. Personen konnten also beispielsweise angeben, dass sie sowohl an einer Bürgerinitiative teilgenommen als auch bei einer Unterschriftensammlung unterschrieben haben. Ein politisches Amt kann im Rahmen einer freiwilligen Tätigkeit übernommen worden sein, es kann sich aber auch um die bezahlte Tätigkeit einer Berufspolitikerin oder eines Berufspolitikers handeln. Im Freiwilligensurvey ist den Befragten überlassen, einzuschätzen, ob sie auch eine berufliche Tätigkeit als Übernahme eines politischen Amtes angeben oder nur eine solche ehrenamtliche Tätigkeit, wobei die Zahl der Berufspolitikerinnen und -politiker in Deutschland viel geringer ist als die Zahl der freiwillig Engagierten in politischen Ämtern.
Die Ergebnisse werden im Folgenden zunächst absteigend nach der Häufigkeit der Nennungen präsentiert. Daran anschließend werden die Ergebnisse kumuliert berichtet, um darzustellen, über wie viele Formen sich Personen politisch beteiligen. Da es sich um eine Retrospektivfrage handelt („Haben Sie schon einmal...?"), in der obwohl es nicht explizit genannt ist, ein Zeitfenster festgelegt ist, dass jeweils die gelebten Jahre umfasst, interpretieren wir die Ergebnisse dergestalt, dass die Befragten jeweils über ihren gesamten bisherigen Lebenslauf betrachtet Auskunft geben. Somit haben ältere Befragte allein aufgrund der Zahl ihrer Lebensjahre eine höhere Wahrscheinlichkeit, die eine oder andere Form der politischen Partizipation ausgeübt $\mathrm{zu}$ haben. Eine grundsätzliche Mindestaltersgrenze für die politische Partizipation existiert nicht, es ist jedoch davon auszugehen, dass politisches Interesse bereits in der Jugend und frühen Erwachsenenphase entsteht, was sich auch in der Diskussion um eine mögliche Absenkung des Wahlalters von derzeit in der Regel 18 Jahren auf 16 Jahre zeigt (derzeit liegt das Wahlalter nur bei Landtagswahlen in Brandenburg und Bürgerschaftswahlen in Bremen bei 16 Jahren, sowie bei einigen Kommunalwahlen, nicht aber bei der Bundestagswahl). Für die Interpretation der Ergebnisse in diesem Kapitel ist von Bedeutung, dass ein längeres Zeitfenster wie in der Retrospektivbefragung grundsätzlich $\mathrm{zu}$ höheren Anteilen führt als ein kürzeres Zeitfenster, wie etwa die letzten zwölf Monate, das bei der Erhebung des freiwilligen Engagements oder aber der Geldspenden im Freiwilligensurvey angewendet wird.

\subsection{Beteiligung an Unterschriftensammlungen und Online-Petitionen}

In Deutschland können Menschen regelmäßig ihren politischen Willen äußern, etwa indem sie eine Unterschriftensammlung beziehungsweise eine Online-Petition für oder gegen eine Sache unterzeichnen (Jungherr \& Jürgens 2011). Die Unterschriftensammlung ist von den hier un- 
tersuchten Partizipationsformen die mit 43,4 Prozent am häufigsten in der Wohnbevölkerung Deutschlands verbreitete Form der Beteiligung (Abbildung 6-1a). Die technische Verfügbarkeit von Online-Plattformen erleichtert zudem, so- wohl eine Unterschriftensammlung zu initiieren als auch an einer solchen teilzunehmen, besonders im Vergleich zum Erstellen und Einsammeln von Papierlisten.

Abbildung 6-1: Anteile derer, die sich schon einmal an einer Unterschriftensammlung oder einer

Online-Petition beteiligt haben, 2014, a) gesamt, nach Geschlecht und nach Alter, b) nach Bildung

a) gesamt, nach Geschlecht und nach Alter

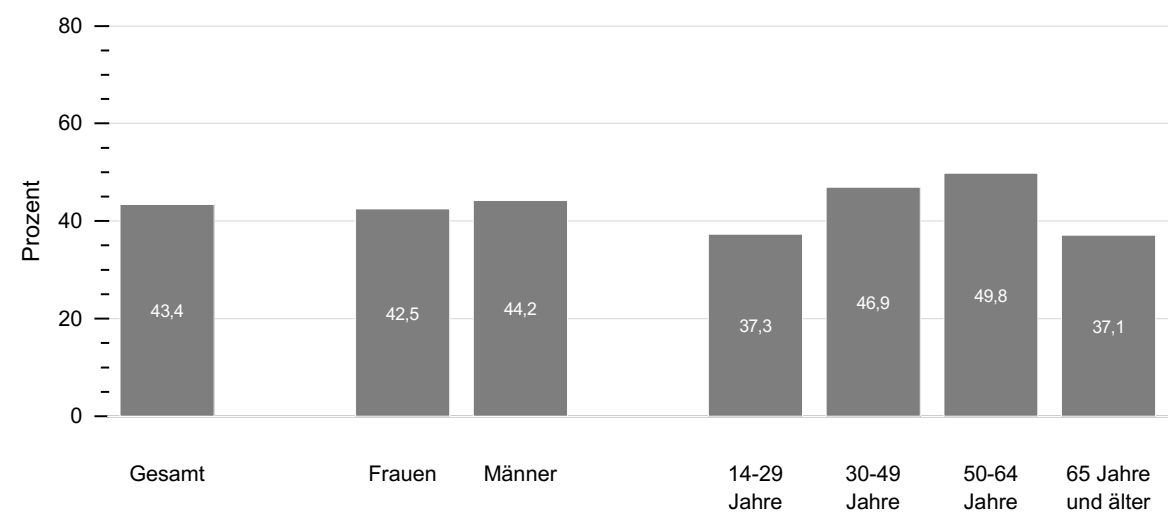

b) nach Bildung

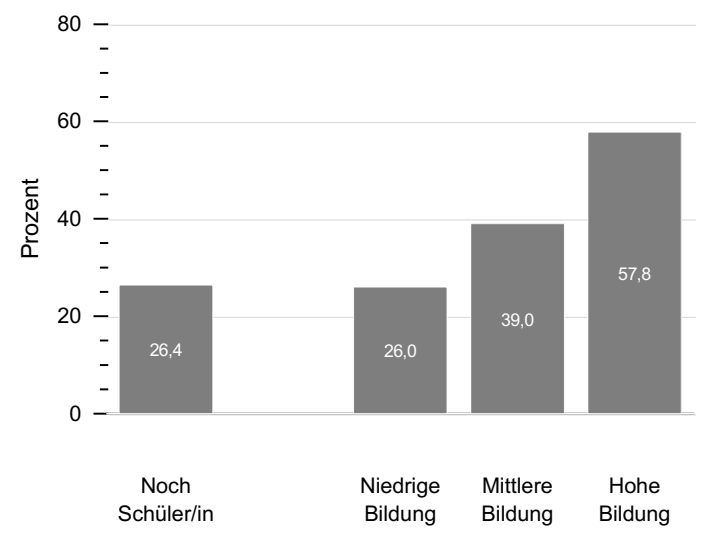

Quelle: FWS 2014, gewichtet, eigene Berechnungen (DZA). Basis: Alle Befragten ( $n=28.547)$.

Von den Männern haben 44,2 Prozent mindestens einmal bei einer Unterschriftensammlung unterschrieben oder eine Online-Petition un- terzeichnet, bei den Frauen sind es 42,5 Prozent. Der Geschlechterunterschied ist bei dieser Form der politischen Partizipation, die lediglich einen 
geringen Zeiteinsatz für Information, Meinungsbildung und Unterschrift erfordert, nur sehr gering ausgeprägt, aber statistisch signifikant. Deutlich größere und ebenfalls signifikante Unterschiede zeigen sich zwischen den Altersgruppen: Anteilig am häufigsten haben die 50- bis 64-Jährigen bei einer Unterschriftensammlung mitgemacht, jede beziehungsweise jeder Zweite dieser Altersgruppe hat sich an einer solchen in der Vergangenheit schon einmal beteiligt - und nicht die Personen im Alter von 65 und mehr Jahren, bei denen man aufgrund der Zahl ihrer Lebensjahre einen noch höheren Anteil erwarten könnte: Mit 37,1 Prozent ist der Anteil bei der ältesten Altersgruppe sogar unterdurchschnittlich. Dies könnte auch daran liegen, dass diese Form der politischen Partizipation in Deutschland erst seit Ende der 1950er Jahre an Bedeutung gewonnen hat, im Zuge der sogenannten partizipatori- schen Revolution (Weßels 2013). Von den 30- bis 49-Jährigen haben bereits 46,9 Prozent schon einmal an einer Unterschriftensammlung teilgenommen, von den 14- bis 29-Jährigen waren es bisher 37,3 Prozent.

Personen mit hoher Bildung beteiligen sich darüber hinaus viel häufiger als Personen mit niedriger und mit mittlerer Bildung an Unterschriftensammlungen beziehungsweise Online-Petitionen. Von den Personen mit niedriger Bildung haben lediglich 26,0 Prozent teilgenommen, von den Personen mit mittlerer Bildung 39,0 Prozent und von den Personen mit hoher Bildung 57,8 Prozent (Abbildung 6-1b). Die Bildungsunterschiede sind also, wie auch in anderen Studien belegt, wesentlich größer als etwa die Unterschiede zwischen den Altersgruppen (vgl. Böhnke 2011; Jörke 2011). 


\subsection{Beteiligung an Demonstrationen}

Neben der Interessensbekundung durch Unterschriftensammlung ist die Teilnahme an einer Demonstration eine Möglichkeit, die eigene Position für oder gegen eine Sache deutlich zu machen. Etwa ein Drittel der Wohnbevölkerung ab 14 Jahren hat schon einmal an einer Demonstration teilgenommen (Abbildung 6-2a). Bei den Frauen ist der Anteil mit 29,2 Prozent insgesamt etwas niedriger als bei den Männern mit 35,3 Prozent. Ein solcher Geschlechterunterschied hinsichtlich der Beteiligung an Demonstrationen findet sich in allen Altersgruppen mit Ausnahme der jüngsten: Hier sind die Teilnahmeraten für Frauen (27,2 Prozent) signifikant höher als für Männer (26,2 Prozent; Tabelle 6-2 im OnlineAnhang).

Wie bei der Unterschriftensammlung ist auch bei der Demonstrationsteilnahme die Altersgruppe der 50- bis 64-Jährigen diejenige mit dem höchsten Anteil bislang politisch aktiver Personen: 39,0 Prozent dieser Altersgruppe ha- ben schon einmal an einer Demonstration teilgenommen. Bei den 30- bis 49-Jährigen waren es bereits 34,9 Prozent und bei den Personen im Alter von 65 und mehr Jahren lediglich 26,1 Prozent. Die größten Unterschiede zeigen sich wiederum zwischen den Bildungsgruppen: Von den Personen mit niedriger Bildung haben lediglich 18,4 Prozent schon einmal an einer Demonstration teilgenommen, von den Personen mit hoher Bildung waren es jedoch 45,2 Prozent (Abbildung 6-2b). Obwohl Personen anteilig insgesamt häufiger bei Unterschriftensammlungen mitmachen als bei Demonstrationen - Letztere sind an konkrete Orte und Termine gebunden und somit weniger flexibel für Teilnehmerinnen und Teilnehmer -, handelt es sich um vergleichbare Phänomene der politischen Partizipation in Form der demonstrativen Demokratie, bei denen die eigene Meinung geäußert wird. Zudem weisen die Unterschiede in den Anteilen zwischen den Bevölkerungsgruppen dieselben Muster auf.

Abbildung 6-2: Anteile derer, die schon einmal an einer Demonstration teilgenommen haben, 2014, a) gesamt, nach Geschlecht und nach Alter, b) nach Bildung

a) gesamt, nach Geschlecht und nach Alter

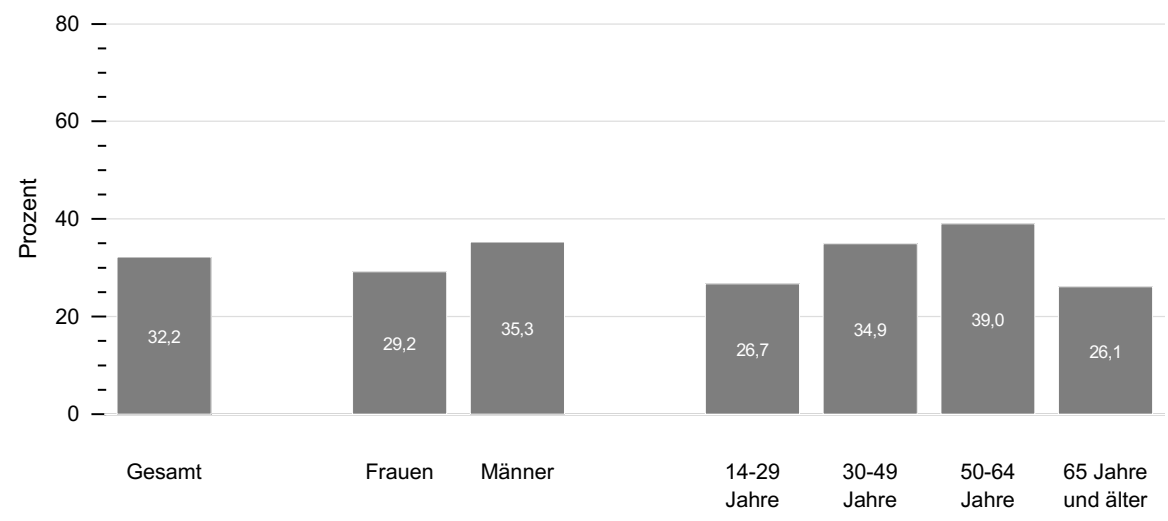




\section{b) nach Bildung}

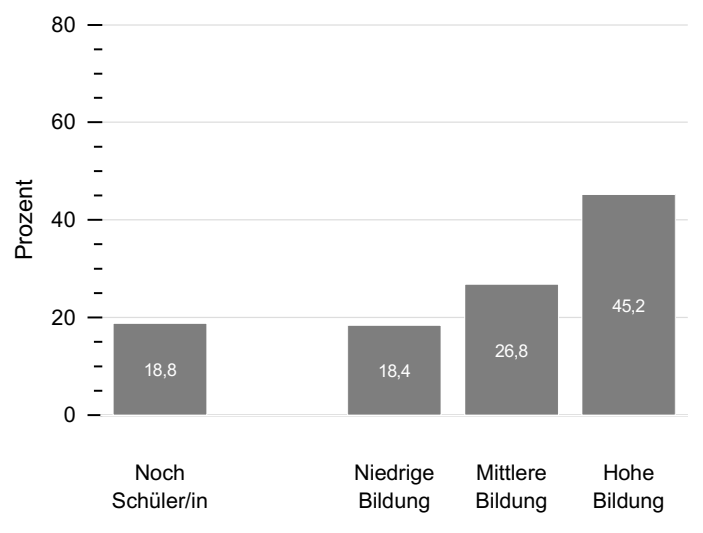

Quelle: FWS 2014, gewichtet, eigene Berechnungen (DZA). Basis: Alle Befragten $(n=28.648)$. 


\subsection{Beteiligung an Bürgerinitiativen}

Bei einer Bürgerinitiative machen Menschen mit, die ein konkretes Ziel unterstützen, beispielsweise um den Bau eines Bahnhofs oder den Abriss eines Baudenkmals zu verhindern, oder etwa den Schutz und den Erhalt einer sozialen Einrichtung zu erreichen. Eines der bekanntesten Beispiele aus jüngerer Zeit sind sicherlich die vielen Initiativen, die gegen den Umbau des Stuttgarter Hauptbahnhofs ,Stuttgart 21' gerichtet sind, wie die Bürgerinitiative ,Leben in Stuttgart $^{\text {'6 }}$ (Gabriel, Schoen \& Faden-Kuhne 2014). Über das konkrete Ziel hinaus lassen sich Bürgerinitiativen dadurch charakterisieren, dass sie kurzfristig eine große Zahl von Anhängerinnen und Anhängern mobilisieren, und sie nach ge- fällten Entscheidungen, unabhängig ob im Einklang mit den Zielen der Initiative oder nicht, von den Organisatoren meist zügig wieder aufgelöst werden. Allerdings gibt es mit längerfristig bestehenden Bürgerinitiativen auch Ausnahmen, die ein überregionales politisches Programm verfolgen wie die Anti-Atomkraft-Bewegung (Pötzsch 2009). Häufig gibt die persönliche Betroffenheit Anlass, eine Bürgerinitiative zu gründen oder auch zu unterstützen (Trumann 2013). An einer Bürgerinitiative haben sich bereits 22,5 Prozent der Wohnbevölkerung Deutschlands ab 14 Jahren beteiligt; wobei sich die Anteile von Frauen und Männern nicht signifikant voneinander unterscheiden (Abbildung 6-3a).

Abbildung 6-3: Anteile derer, die sich schon einmal an einer Bürgerinitiative beteiligt haben, 2014, a) gesamt, nach Geschlecht und nach Alter, b) nach Bildung

a) gesamt, nach Geschlecht und nach Alter

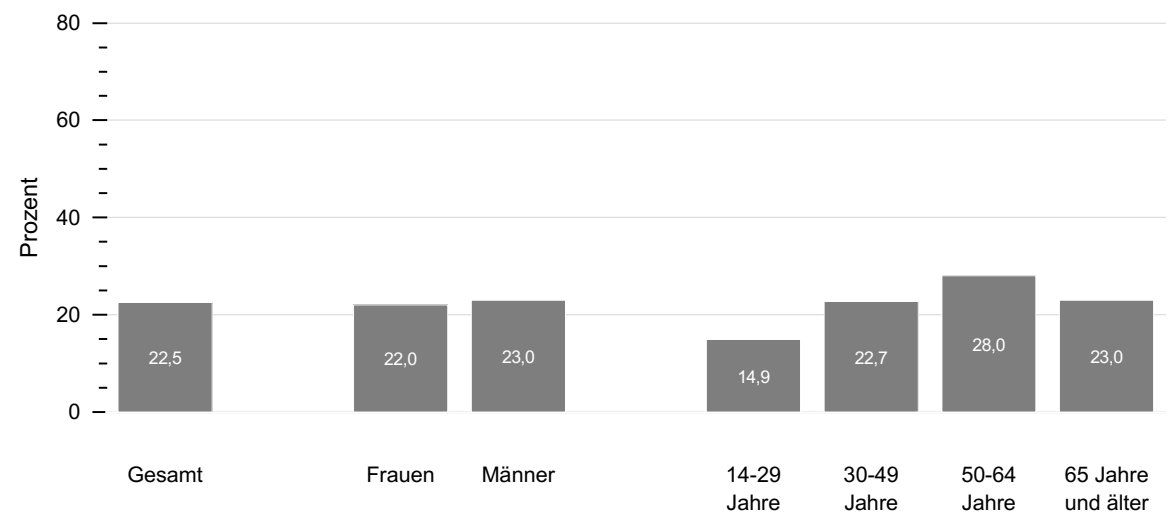

6 www.leben-in-stuttgart.de 


\section{b) nach Bildung}

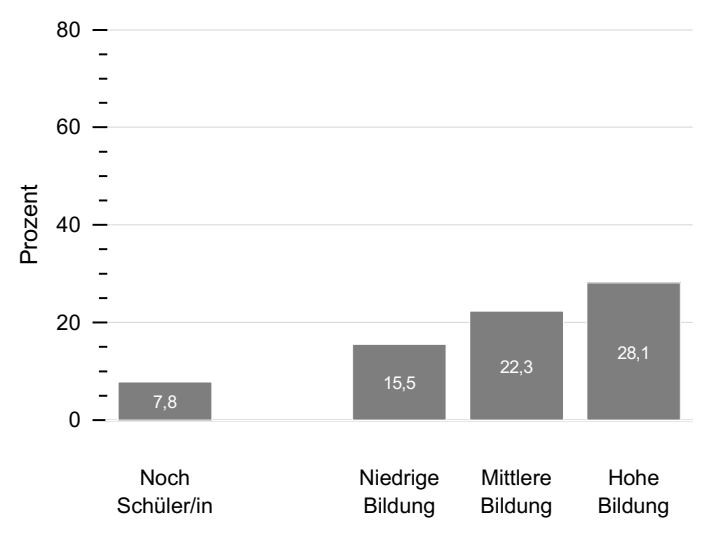

Quelle: FWS 2014, gewichtet, eigene Berechnungen (DZA). Basis: Alle Befragten ( $n=28.573$ ).

Wie bei den anderen betrachteten Formen der politischen Partizipation sind es auch hier die 50- bis 64-Jährigen, die sich über ihren bisherigen Lebenslauf betrachtet anteilig am häufigsten beteiligt haben; 28,0 Prozent in dieser Altersgruppe haben in der Vergangenheit bei einer Bürgerinitiative mitgemacht beziehungsweise machen derzeit dort mit. In den anderen Altersgruppen ist der Anteil geringer, am niedrigsten ist der Anteil bei den 14- bis 29-Jährigen, von denen bislang lediglich 14,9 Prozent an einer Bürgerinitiative beteiligt waren. Wiederum zeigen sich die größten Unterschiede zwischen den Bildungsgruppen. Während von den Personen mit niedriger Bildung 15,5 Prozent bei einer Initiative mitgemacht haben, waren es bei Personen mit hoher Bildung 28,1 Prozent (Abbildung 6-3b). Diese Bildungsdifferenzen zeigen sich in allen Altersgruppen und sowohl bei Frauen als auch bei Männern, der Unterschied ist allerdings bei Frauen etwas stärker ausgeprägt. Während die Anteile derer, die schon einmal bei einer Bürgerinitiative mitgemacht haben, bei höhergebildeten Frauen (28,4 Prozent) und Männern (27,8 Prozent) nahezu gleich sind, unterscheiden sich die geschlechterspezifischen Raten bei den Niedriggebildeten deutlicher: Frauen dieser Bildungsgruppe haben sich zu 14,0 Prozent bereits an einer Bürgerinitiative beteiligt, Männer zu 17,1 Prozent. Das bedeutet: Die Differenz in der Beteiligung zwischen niedrig- und hochgebildeten Männern beträgt 10,7 Prozentpunkte, bei den Frauen liegt sie mit 14,4 Prozentpunkten noch etwas darüber (siehe Tabelle 6-3 im OnlineAnhang). Somit haben Personen mit niedriger Bildung weniger Möglichkeiten, ihre politischen Standpunkte zu bekunden und an Planungs- und Entscheidungsprozessen mitzuwirken. 


\section{6 Übernahme politischer Ämter}

Politische Ämter zu übernehmen ist eine Form der politischen Partizipation mit einem hohen Verpflichtungsgrad. An dieser Stelle betrachten wir, wer in der Vergangenheit diese Form der politischen Partizipation ausgeübt hat beziehungsweise diese derzeit ausübt. Dabei handelt es sich anders als bei den bisher betrachteten Partizipationsformen in der Regel um eine Art des freiwilligen Engagements (wenn das Amt ehrenamtlich ausgeübt wird) oder aber um eine bezahlte Tätigkeit. Politische Ämter sind vielleicht am bekanntesten auf der Ebene der Bundes- und Landespolitik, wo sie meist von Berufspolitikerinnen und -politikern ausgeübt werden und mit prominenten Gesichtern wie Bundeskanzlerin oder Bundeskanzler und Ministerpräsidentinnen und -präsidenten in Verbindung gebracht werden. Am häufigsten werden politische Ämter jedoch in der Kommunalpolitik und zwar in der Regel als Ehrenamt ausgeübt (Reiser 2011). Beispiele für öffentliche Ämter sind hier das Bürgermeister- sowie das Abgeordnetenamt in Stadtparlamenten oder Kreistagen. Naßmacher (2011) betont die besondere Bedeutung der kommunalen Ebene, die unter anderem darin besteht, dass die Kommunen Orte der Elitesozialisation sind. Das heißt, hier werden Personen erstmalig für politische Ämter ausgewählt und an die Übernahme weiterer Aufgaben herangeführt. Gleichzeitig weist Naßmacher darauf hin, dass es schwierig ist, genügend geeignete Personen $\mathrm{zu}$ finden, die bereit sind, etwa die Arbeit in den Gemeinderäten zu übernehmen. Schließlich müssten diese auch jede Menge Kritik durch die Bürgerinnen und Bürger einstecken können, denn heute würden die Entscheidungen der Gewählten immer stärker kritisiert werden (Naßmacher 2011: 12).

Von der Wohnbevölkerung im Alter von 14 und mehr Jahren in Deutschland hat ein Anteil von 8,7 Prozent mindestens einmal im Leben ein politisches Amt übernommen. Bei den Frauen ist der Anteil mit 6,8 Prozent kleiner als bei den Männern mit 10,8 Prozent (Abbildung 6-4a). Männer sind somit vergleichsweise häufiger im
Rahmen eines politischen Amtes tätig als Frauen. In einer aktuellen Studie über Kommunalpolitikerinnen, die im Auftrag des Bundesministeriums für Familien, Senioren, Frauen und Jugend durchgeführt wurde, wird ein solcher Geschlechterunterschied ebenfalls festgestellt (Kletzing \& Lukoschat 2014). Insbesondere vor dem Gebot der Gleichstellung ist ein solcher Unterschied kritisch zu diskutieren.

Bezüglich der Übernahme eines politischen Amtes zeigen sich zudem zwischen den Altersgruppen deutliche Unterschiede, wobei Personen im Alter von 65 und mehr Jahren bereits anteilig am häufigsten ein politisches Amt ausgefüllt haben: 12,8 Prozent dieser Altersgruppe und 11,7 Prozent der 50- bis 64-Jährigen haben schon einmal ein politisches Amt ausgeübt oder anderweitig politische Verantwortung übernommen. Die entsprechenden Anteile bei den jüngeren Altersgruppen sind mit 4,7 Prozent der 14- bis 29-Jährigen und mit 5,9 Prozent der 30- bis 49-Jährigen deutlich geringer. In der Tat gelten für einige politische Ämter nach wie vor Mindestaltersgrenzen. Das wohl prominenteste Beispiel ist die im Grundgesetz festgelegte Mindestaltersgrenze von 40 Jahren für die Wahl zur Bundespräsidentin beziehungsweise zum Bundespräsidenten. Für die meisten Ämter auf zum Beispiel kommunaler und Bundesebene gelten dagegen verschiedene Altersgrenzen, teils ist ein Mindestalter von 23 Jahren (etwa für das Bürgermeisteramt in Niedersachsen), von 25 Jahren (etwa für das Bürgermeisteramt in Brandenburg) oder 27 Jahren (etwa für das Bürgermeisteramt in Schleswig-Holstein) vorgesehen. Im Übrigen sind in den Gemeindeordnungen auch häufig Höchstaltersgrenzen für diese Ämter festgeschrieben, in Baden-Württemberg, Bayern und Rheinland-Pfalz liegt diese beispielsweise bei 65 Jahren. Über die Notwendigkeit zur Neuregelung (im Sinne einer Verschiebung der Höchstaltersgrenzen nach oben oder einer Flexibilisierung beziehungsweise Abschaffung der Altersgrenzen) wird in vielen Bereichen der politischen 
Ämter, aber auch der Ehrenämter diskutiert. Einerseits haben Altersgrenzen strukturierende Funktionen, die für Kommunen und Individuen von Vorteil sein können, andererseits sind sie möglicherweise zu starr und altersdiskriminierend (Zeman 2010).

Abbildung 6-4: Anteile derer, die schon einmal ein politisches Amt übernommen haben, 2014, a) gesamt, nach Geschlecht und nach Alter, b) nach Bildung

a) gesamt, nach Geschlecht und nach Alter

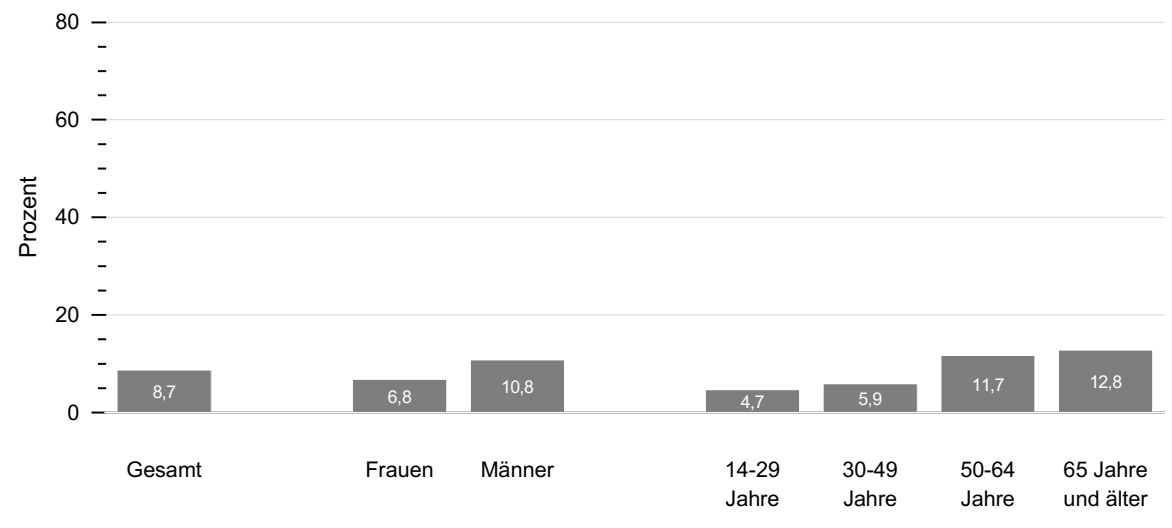

b) nach Bildung

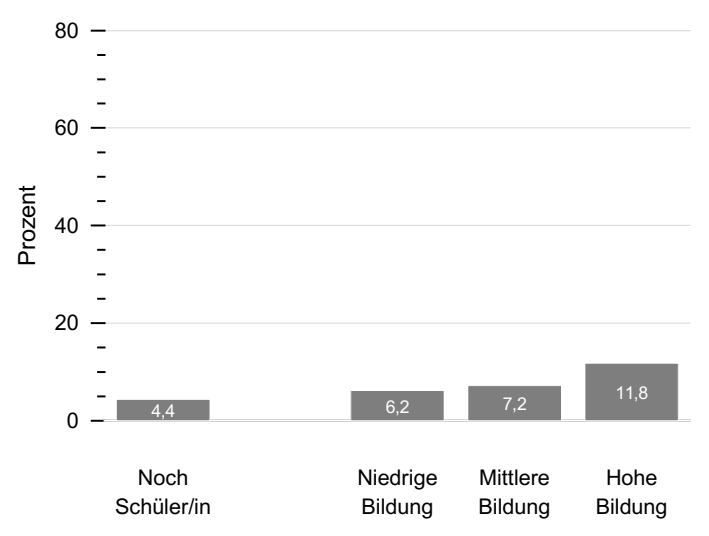

Quelle: FWS 2014, gewichtet, eigene Berechnungen (DZA). Basis: Alle Befragten $(n=28.652)$.

Bildungsunterschiede, die sich schon bezüglich des Mitmachens bei der politischen Meinungsbekundung zeigten, wirken bei der Ausübung eines konkreten politischen Amtes fort: Während 11,8
Prozent der Personen mit hoher Bildung schon einmal ein politisches Amt übernommen haben, sind es bei den Personen mit niedriger Bildung lediglich 6,2 Prozent. Am kleinsten ist der Anteil 
mit 4,4 Prozent bei den Schülerinnen und Schülern, was mit dem geringen Anteil in der jüngsten Altersgruppe korrespondiert. Durch ihr jüngeres Lebensalter hatten Schülerinnen und Schüler bislang weniger Gelegenheiten, politische Ver- antwortung zu übernehmen. Zu berücksichtigen ist außerdem, dass für politische Ämter im engeren Sinne in der Regel ein Mindestalter von 18 Jahren gilt, das die meisten der Schülerinnen und Schüler noch nicht erreicht haben.

\subsection{Politisch partizipieren in verschiedenen Formen}

Betrachtet man die vier untersuchten Partizipationsformen gemeinsam, zeigt sich, dass sich eine Mehrheit aller in Deutschland lebenden Personen im Alter ab 14 Jahren auf mindestens eine Art beteiligt hat. Von allen Personen im Alter ab 14 Jahren haben sich 58,2 Prozent in mindestens einer der vier hier untersuchten Formen politisch beteiligt (Abbildung 6-5a). Ein Viertel hat sich lediglich in einer Form beteiligt. Etwa ein Drittel aller Personen hat sogar schon auf verschiedene Arten und Weisen politisch partizipiert, mehr als jede beziehungsweise jeder Achte hat sogar drei und mehr Partizipationsformen genannt.
Gruppenspezifisch zeigen sich hier ganz ähnliche Partizipationsmuster wie schon in der Einzelbetrachtung: Frauen partizipieren seltener überhaupt und seltener in mehreren Formen als Männer. Hinsichtlich des Alters zeigen sich die höchsten Partizipationsraten bei Personen zwischen 50 und 64 Jahren. Die niedrigsten Partizipationsraten zeigen sich aufgrund ihres noch jungen Alters bei den Schülerinnen und Schülern, aber auch Niedriggebildete partizipieren $\mathrm{zu}$ wesentlich geringeren Anteilen überhaupt und in unterschiedlichen Formen als Personen mit mittlerer Bildung und - noch deutlicher - als Hochgebildete.

Abbildung 6-5: Anteile derer, die sich in einer oder mehreren politischen Form(en) beteiligt haben, 2014, a) gesamt, nach Geschlecht und nach Alter, b) nach Bildung

a) gesamt, nach Geschlecht und nach Alter

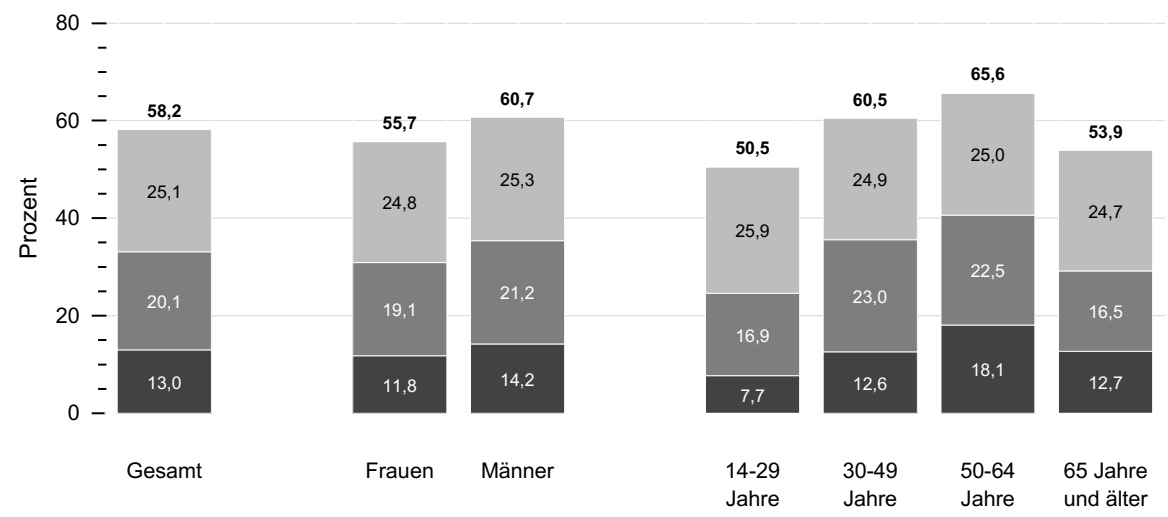


b) nach Bildung

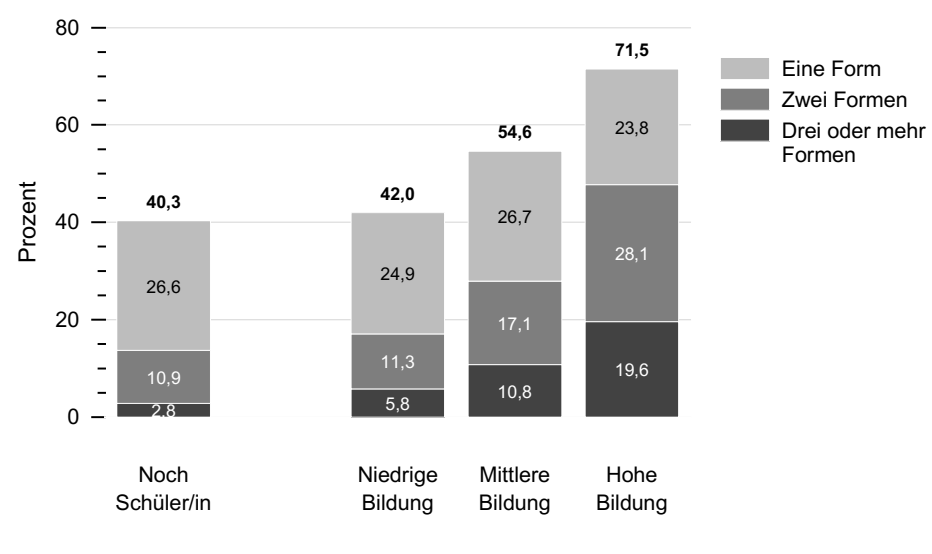

Quelle: FWS 2014, gewichtet, eigene Berechnungen (DZA). Basis: Alle Befragten $(n=28.385)$.

\subsection{Fazit}

Was bedeuten die gezeigten Befunde für die Demokratie? Zunächst einmal ist festzustellen, dass Bürgerinnen und Bürger über verschiedene Wege am politischen Leben teilnehmen. Verbreitete Formen sind - wenn man die Beteiligung über den gesamten bisherigen Lebenslauf betrachtet: die Teilnahme an Unterschriftensammlungen, an Demonstrationen und an Bürgerinitiativen. Diese hier betrachteten Formen sind weit verbreitet, allerdings nicht so verbreitet wie die Beteiligung an Wahlen. So lag die Wahlbeteiligung bei der Bundestagswahl 2013 laut Bundeswahlleiter bei 71,5 Prozent. Am seltensten übernehmen Menschen dagegen selbst politische Ämter.

Insgesamt haben sich bereits 58,2 Prozent der Wohnbevölkerung ab 14 Jahren in mindestens einer der vier hier untersuchten Formen politisch beteiligt. Dies bedeutet aber auch, dass sich mehr als zwei Fünftel bislang weder über Bürgerinitiativen, noch über Unterschriftensammlungen, Demonstrationen oder die Übernahme eines Amtes am politischen Leben beteiligt haben.
Die Altersgruppe der 50- bis 64-Jährigen hat die höchsten Beteiligungsquoten an Unterschriftensammlungen, Demonstrationen und Bürgerinitiativen. 11,7 Prozent dieser Altersgruppe und 12,8 Prozent der Personen im Alter von 65 und mehr Jahren haben schon einmal ein politisches Amt ausgeübt oder anderweitig politische Verantwortung übernommen. Die entsprechenden Anteile bei den jüngeren Altersgruppen sind deutlich geringer. Allerdings wissen wir durch die Retrospektivbefragung nicht, wie alt die befragten Personen tatsächlich waren, als sie dergestalt partizipierten, und auch nicht, ob sie nur einmal oder über einen längeren Zeitraum in ihrem Lebenslauf politisch aktiv waren. Es lässt sich hier lediglich schlussfolgern, dass die 50- bis 64-Jährigen in ihrem bisherigen Leben aktiver waren als die jüngeren Altersgruppen. Allerdings ist eine mögliche gleich hohe Beteiligung der heutigen Jüngeren in Zukunft davon unbenommen.

Frauen beteiligen sich zu geringeren Anteilen politisch als Männer. Große Differenzen finden sich bei der Teilnahme an Demonstrationen und bei der Übernahme politischer Ämter. Dies 
kann auf unterschiedliche Faktoren zurückzuführen sein (Kletzing \& Lukoschat 2014): Zum einen können für alle Formen der politischen Partizipation Effekte geschlechtsspezifischer Sozialisation bedeutsam sein (,Sozialisationsthe-

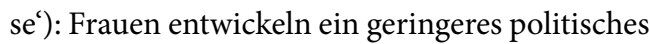
Interesse als Männer, da unsere politische Kultur und unsere politischen Institutionen männlich dominiert sind. Zum anderen unterscheiden sich die strukturellen Voraussetzungen und Ressourcen von Frauen und Männern nach wie vor häufig. Frauen verfügen zum Beispiel über im Durchschnitt schlechtere berufliche Positionen und damit verbundene Kontakte, um ein politisches Amt zu übernehmen als Männer (,Sozialstrukturthese'). Darüber hinaus haben Frauen aufgrund der geschlechtsspezifischen Arbeitsteilung ein geringeres Zeitbudget für politische Ämter und andere Formen politischer Beteiligung als Männer (,Abkömmlichkeitsthese'). Schließlich können Diskriminierungsprozesse eine Rolle spielen, beispielsweise wenn Frauen bei der Rekrutierung für politische Ämter benachteiligt werden (,Diskriminierungsthese') oder sie bei Wahlen für politische Ämter geringere Chancen haben als Männer (,Wahlbenachteiligungsthese').

Lediglich bei der Beteiligung in Bürgerinitiativen sind die Partizipationsraten von Frauen und Männern gleich. Dies könnte damit zu erklären sein, dass für die Beteiligung an Bürgerinitiativen häufig die lokale Betroffenheit durch einen Sachverhalt sowie die zeitliche Begrenztheit ausschlaggebend ist. Diese ist für Frauen und Männer in der Regel gleichermaßen gegeben, und könnte zumindest kurzfristig etwa geschlechtsspezifische Effekte der beruflichen Netzwerke und der Rekrutierung für politische Ämter überschreiben. Von einer langfristigen Angleichung der politischen Beteiligung zwischen Frauen und Männer ist allerdings nicht auszugehen, da bei den jüngeren Altersgruppen Geschlechterunterschiede ebenso zu beobachten sind wie bei den älteren. Zudem ist insbesondere in Bezug auf berufliche Karrieren auch auf die Wechselwirkungen zwischen den Lebensbereichen hinzuweisen, so kann die erfolgreiche Ausübung eines politischen Amtes nicht nur eine berufliche Erfahrung voraussetzen, sondern eben auch zu beruflichen Erfolgen in anderen Bereichen beitragen und die Geschlechterungleichheit erhöhen. Die unterdurchschnittlichen politischen Partizipationschancen der Frauen werden übrigens auch anhand der Engagementquoten im Bereich Politik und politische Interessenvertretung deutlich. Mit fünf Prozent sind Männer anteilig doppelt so häufig in diesem Bereich freiwillig engagiert wie Frauen (siehe Kapitel 3).

Besonders auffällig sind die starken Bildungsunterschiede: Alle Partizipationsformen sind bei Personen mit hoher Schulbildung weiter verbreitet als bei jenen mit mittlerer oder niedriger Bildung. Während sich beispielsweise mehr als die Hälfte der Personen mit hoher Bildung schon einmal an einer Unterschriftensammlung beteiligt hat, hat dies von den Personen mit niedriger Bildung nur etwa jede beziehungsweise jeder Vierte schon einmal getan. Ein politisches Amt hat etwa jede achte Person mit hoher Bildung schon einmal ausgeübt, bei den Niedriggebildeten ist es nur jede dreiundzwanzigste Person. Es zeigt sich somit einmal mehr, dass die politische Beteiligung an Voraussetzungen geknüpft ist. Neben dem Interesse für politische Belange sind die Überzeugung notwendig, selbst etwas politisch verändern zu können, sowie tatsächliche Möglichkeiten zur Mitsprache und Einflussnahme. Diese variieren stark mit dem sozialen Status einer Person, wie die nach Bildungsniveaus aufgeschlüsselten Ergebnisse zeigen. Genau aus diesem Grund werden insbesondere Bürgerinitiativen als Form der direkten Demokratie beziehungsweise der deliberativen Demokratie teils auch kritisiert: Sie rekrutieren mehrheitlich Personen aus der oberen Mittelschicht, gleichzeitig vertreten sie teilweise lokale Minderheitsinteressen gegen Mehrheitsinteressen und beanspruchen Entscheidungskompetenzen, die eigentlich nur demokratisch gewählten Vertreterinnen und Vertretern zukommen (Pötzsch 2009). Die Interessen von Personen mit niedriger Bildung werden bei solchen direktdemokratischen Verfahren 
möglicherweise nicht gleichberechtigt gehört und berücksichtigt (Geißel 2015). Dies ist insbesondere deshalb diskussionswürdig, weil die Intention einer verbesserten Bürgerbeteiligung durchaus in der Stärkung der Demokratie liegt, der nichtintendierte Nebeneffekt einer Verschärfung der sozialen Ungleichheit in der politischen Partizipation jedoch auch das Gegenteil bewirken könnte (Schäfer \& Schoen 2013).

Unter dem Gesichtspunkt des Grundsatzes politischer Gleichheit erscheinen diese ungleichen Partizipationsraten durchaus problematisch: „Die ungleiche Teilhabe an politischen Prozessen untergräbt das demokratische Ideal politischer Gleichheit, das die gleiche Berücksichtigung von Interessen der Bevölkerung fordert" (Bödeker 2012a: 6). Beteiligen sich dagegen nur ausgewählte Bevölkerungsgruppen wie Höhergebildete aus der oberen Mittelschicht, so ist die Wahrscheinlichkeit groß, dass deren Interessen stärker in politischen Reformen berücksichtigt werden, als die derjenigen, die sich weniger stark in politische Prozesse einbringen. Es ist daher zu überlegen, wie die politische Beteiligung gerade von Personen mit geringen sozioökonomischen Ressourcen besser gefördert werden kann, etwa die Beteiligung von Frauen mit geringer Bildung. Sinnvoll sind hier sicherlich das frühzeitige Stärken des politischen Interesses und das Erlernen politischer Zusammenhänge und Hintergründe im Rahmen von Schule und Ausbildung. Darüber hinaus scheint es wichtig, Barrieren zur politischen Partizipation für benachteiligte Gruppen abzubauen. Die stärkere Einbeziehung von Bürgerinnen und Bürgern in kommunale und lokale politische Planungs- und Entscheidungsprozesse könnte das politische Interesse auch bislang eher politikferner Personen sowie die Überzeugung, selbst an politischen Entscheidungen mitwirken zu können, befördern.

\section{Literatur}

Bödeker, S. (2012a). Soziale Ungleichheit und politische Partizipation in Deutschland. WZBrief Zivilengagement, 5, 2-7.

Bödeker, S. (2012b). Soziale Ungleichheit und politische Partizipation in Deutschland. Grenzen politischer Gleichheit in der Bürgergesellschaft. Frankfurt am Main: Otto Brenner Stiftung.

Böhnke, P. (2011). Ungleiche Verteilung politischer und zivilgesellschaftlicher Partizipation. Aus Politik und Zeitgeschichte, 61(1/2), 18-25.

Cornelissen, W. (1993). Politische Partizipation von Frauen in der alten Bundesrepublik und im vereinten Deutschland. In: G. Helwig \& H. M. Nickel (Hrsg.) Frauen in Deutschland 1945-1992 (S. 321-350). Berlin: Akademie-Verlag.

Deth, J. W. van (2009). Politische Partizipation. In: V. Kaina \& A. Römmele (Hrsg.) Politische Soziologie (S. 142-161). Wiesbaden: VS Verlag für Sozialwissenschaften.

Gabriel, O. (2014). Bürgerbeteiligung in Deutschland. In: K. Pohl \& P. Massing (Hrsg.) Mehr Partizipation - mehr Demokratie? (S. 27-45). Schwalbach/Taunus: Wochenschau Verlag.

Gabriel, O. W., Schoen, H., \& Faden-Kuhne, K. (2014). Der Volksentscheid über Stuttgart 21. Aufbruch zu neuen demokratischen Ufern? Opladen: Barbara Budrich.

Geißel, B. (2015). Mitwirkung für alle? Thesen zur politischen Ungleichheit. In: L. Harles \& D. Lange (Hrsg.) Zeitalter der Partizipation. Paradigmenwechsel in Politik und politischer Bildung? (S. 37-48). Schwalbach/Taunus: Wochenschau Verlag.

Jörke, D. (2011). Bürgerbeteiligung in der Postdemokratie. Aus Politik und Zeitgeschichte 61(1-2), 13-18.

Jungherr, A., \& Jürgens, P. (2011). E-Petitionen in Deutschland: Zwischen niedrigschwelligem Partizipationsangebot und quasi-plebiszitärer Nutzung. Zeitschrift für Parlamentsfragen, 3, 521-534.

Kersting, N. (2013). Wutbürger und andere soziale Bewegungen. Der Bürger im Staat, 65(3), 155-165.

Kletzing, U., \& Lukoschat, H. (2014). Engagiert vor Ort - Wege und Erfahrungen von Kommunalpolitikerinnen. Berlin: Bundesministerium für Familie, Senioren, Frauen und Jugend. 
Naßmacher, H. (2011). Kommunalpolitik in Deutschland. Aus Politik und Zeitgeschichte 61(7-8), 6-12.

Pötzsch, H. (2009). Die deutsche Demokratie. Bonn: Bundeszentrale für politische Bildung.

Reiser, M. (2011). Kommunalpolitisches Ehrenamt. In: T. Olk \& B. Hartnuß (Hrsg.) Handbuch Bürgerschaftliches Engagement (S. 291-303). Weinheim: Beltz Juventa.

Roth, R. (2011). Partizipation. In: T. Olk \& B. Hartnuß (Hrsg.) Handbuch Bürgerschaftliches Engagement (S. 77-88). Weinheim: Beltz Juventa.

Schäfer, A., \& Schoen, H. (2013). Mehr Demokratie, aber nur für wenige? Der Zielkonflikt zwischen mehr Beteiligung und politischer Gleichheit. Leviathan, 41(1), 94-120.

Trumann, J. (2013). Lernen in Bewegung(en). Politische Partizipation und Bildung in Bürgerinitiativen. Bielefeld: transcript.

Wagner, P. (2013). Bürgerinitiative - Betroffenheit - Selbstbestimmung. Die „Partizipatorische Revolution“ in der Bundesrepublik Deutschland der 1970er Jahre und ihre Sprache. Halle (Saale): Martin-Luther-Universität Halle-Wittenberg.

Weßels, B. (2013). Politische Integration und politisches Engagement. In: Statistisches Bundesamt \& Wissenschaftszentrum Berlin für Sozialforschung (Hrsg.) Datenreport 2013. Ein Sozialbericht für die Bundesrepublik Deutschland (S. 363-376). Bonn: Bundeszentrale für politische Bildung.

Westle, B. (2001). Politische Partizipation und Geschlecht. In: A. Koch, M. Wasmer \& P. Schmidt (Hrsg.) Politische Partizipation in der Bundesrepublik Deutschland (S. 131-168). Opladen: Leske + Budrich.

Zeman, P. (2010). Altersgrenzen auf dem Prüfstand. Berlin: Berliner Senatsverwaltung für Integration, Arbeit und Soziales. Landesstelle für Gleichbehandlung - gegen Diskriminierung.

Open Access Dieses Kapitel wird unter der Creative Commons Namensnennung 4.0 International Lizenz (http://creativecommons.org/licenses/by/4.0/deed.de) veröffentlicht, welche die Nutzung, Vervielfältigung, Bearbeitung, Verbreitung und Wiedergabe in jeglichem Medium und Format erlaubt, sofern Sie den/die ursprünglichen Autor(en) und die Quelle ordnungsgemäß nennen, einen Link zur Creative Commons Lizenz beifügen und angeben, ob Änderungen vorgenommen wurden.

Die in diesem Kapitel enthaltenen Bilder und sonstiges Drittmaterial unterliegen ebenfalls der genannten Creative Commons Lizenz, sofern sich aus der Abbildungslegende nichts anderes ergibt. Sofern das betreffende Material nicht unter der genannten Creative Commons Lizenz steht und die betreffende Handlung nicht nach gesetzlichen Vorschriften erlaubt ist, ist für die oben aufgeführten Weiterverwendungen des Materials die Einwilligung des jeweiligen Rechteinhabers einzuholen. 